\title{
A GENERALIZATION OF A THEOREM OF TATCHELL
}

\author{
NIRANJAN SINGH
}

ABstract. Necessary and sufficient conditions for $\sum a_{n} \varepsilon_{n}$ to be summable $\left|A, \lambda_{n}\right|$, whenever $\Sigma a_{n}$ is convergent, have been obtained. The sufficiency part of this result has also been improved.

1. Let $\sum_{n=0}^{\infty} a_{n}$ be a given infinite series and let $\left\{\lambda_{n}\right\}$ be a sequence of nonnegative numbers increasing to infinity. For any $k>0$, we write

$$
A^{k}(t)=\sum_{\lambda_{n}<t}\left(t-\lambda_{n}\right)^{k} a_{n} .
$$

We say that $\sum_{n=0}^{\infty} a_{n}$ is summable $\left(R, \lambda_{n}, k\right)$ to $S$ if $t^{-k} A^{k}(t) \rightarrow S$ as $t \rightarrow \infty$. If, in addition, $t^{-k} A^{k}(t)$ is of bounded variation in $(0, \infty)$, we say that the series $\sum_{n=0}^{\infty} a_{n}$ is summable $\left|R, \lambda_{n}, k\right|$ to $S$. The discontinuous Riesz means $\left(R^{*}, \lambda_{n}, k\right)$ are obtained by restricting $t$ to the sequence $\left\{\lambda_{n}\right\}$. If $\Sigma a_{n} e^{-\lambda_{n} x}$ is convergent for all positive $x$ and $f(x)=\sum a_{n} e^{-\lambda_{n} x} \rightarrow S$ when $x \rightarrow 0$, then we say that the series $\sum_{n=0}^{\infty} a_{n}$ is summable $\left(A, \lambda_{n}\right)$ to sum $S$, and write $\sum_{n=0}^{\infty} a_{n}=$ $S\left(A, \lambda_{n}\right)$. When $\lambda_{n}=n$, the $\left(A, \lambda_{n}\right)$ method is the Abel method. The series $\sum_{n=0}^{\infty} a_{n}$ is said to be absolutely summable $\left(A, \lambda_{n}\right)$ or summable $\left|A, \lambda_{n}\right|$, if the series $\sum_{n=0}^{\infty} a_{n} e^{-\lambda_{n} x}$ is convergent for all positive values of $x$ and the sum function $f(x)=\sum_{n=0}^{\infty} a_{n} e^{-\lambda_{n} x}$ is of bounded variation in $[0, \infty)$. We also define $\Delta \varepsilon_{n}=\varepsilon_{n}-\varepsilon_{n+1}$.

2. Necessary and sufficient conditions on $\left\{\varepsilon_{n}\right\}$ in order that $\sum a_{n} \varepsilon_{n}$ should be absolutely Abel summable whenever $\sum a_{n}$ converges were obtained by Tatchell [3] in 1954 in the form of the following

THEOREM. Necessary and sufficient conditions for $\sum a_{n} \varepsilon_{n}$ to be summable $|A|$, whenever $\sum a_{n}$ is convergent are

$$
\sum\left|\Delta \varepsilon_{n}\right|<\infty
$$

and

$$
\sum\left|\varepsilon_{n}\right| / n<\infty \text {. }
$$

The object of this paper is to generalize the above theorem by replacing absolute Abel summability $|A|$ by summability $\left|A, \lambda_{n}\right|$ for any sequence $\left\{\lambda_{n}\right\}$ satisfying weaker conditions. In what follows we prove the following theorem.

Received by the editors November 30, 1976.

AMS (MOS) subject classifications (1970). Primary 40G10, 46B15.

Key words and phrases. $\left(A, \lambda_{n}\right)$ summability, Banach space, linear functionals and Riesz means. 
THEOREM 1. Necessary and sufficient conditions for $\sum a_{n} \varepsilon_{n}$ to be summable $\left|A, \lambda_{n}\right|$, whenever $\sum a_{n}$ is convergent, are

$$
\begin{gathered}
\sum\left|\Delta \varepsilon_{n}\right|<\infty, \\
\sum\left|\varepsilon_{n}\right|\left(1-\mu_{n}\right)<\infty,
\end{gathered}
$$

where

$$
\mu_{n}=\lambda_{n} / \lambda_{n+1} \text { and } \lambda_{n+1}=O\left(\lambda_{n}\right)
$$

Taking $\lambda_{n}=n$ in our theorem, the above-mentioned theorem of Tatchell follows as a special case.

3. To prove the theorem we need the following lemmas:

LEMMA 1. If $H(p)=\alpha_{p}$ is a transformation from a Banach space $B$ to the space $L$, and if $h_{x}(p)=\alpha_{p}(x)$ is a continuous linear functional on $B$ whenever $x>0$, then $H(p)$ is a bounded linear operator.

$L$ in this context is really $\dot{L}$ as defined in Dunford and Schwartz, Linear operators. I, p. 119.

The proof of this lemma may be found in [4].

LEMMA 2. If a sequence $\left\{\varepsilon_{n}\right\}$ has the property that the function

$$
\sum_{0}^{\infty} S_{n} \varepsilon_{n}\left\{\frac{d}{d x} \Delta e^{-\lambda_{n} x}\right\}
$$

is defined and has a finite Lebesgue integral on $[0, \infty)$, whenever $\left\{S_{n}\right\}$ is a convergent sequence, then there is a number $H$ such that

$$
\int_{0}^{\infty}\left|\sum_{n=0}^{\infty} S_{n} \varepsilon_{n}\left\{\frac{d}{d x} \Delta e^{-\lambda_{n} x}\right\}\right| d x<H \overline{\mathrm{bd}}\left|S_{n}\right|
$$

for every convergent sequence $\left\{S_{n}\right\}$.

Proof. If

$$
h_{x}(S)=\sum_{n=0}^{\infty} S_{n} \varepsilon_{n}\left\{\frac{d}{d x} \Delta e^{-\lambda_{n} x}\right\}
$$

is defined whenever $S=\left\{S_{n}\right\}$ is a convergent sequence, then $h_{x}$ is a linear functional on the Banach space $C$. Therefore, by hypothesis, $h_{x}$ is a linear functional on $C$ whenever $0<x<\infty$.

Also by hypothesis

$$
\sum_{n=0}^{\infty} S_{n} \varepsilon_{n}\left\{\frac{d}{d x} \Delta e^{-\lambda_{n} x}\right\}
$$

is in the space $L$ whenever $S$ is in $C$. Hence the lemma follows from Lemma 1.

LEMMA 3. If a sequence $\left\{p_{n}\right\}$ of elements in a Banach space $B$ has the property that there is a number $H$ such that for every nonnegative integer $K$ and 
every set of real numbers $\theta_{0}, \theta_{1}, \theta_{2}, \ldots, \theta_{k}$,

$$
\left\|\sum_{n=0}^{k} e^{i \theta_{n}} p_{n}\right\|<H
$$

then $\sum_{n=0}^{\infty}\left|f\left(p_{n}\right)\right|<\infty$, for every linear functional $f$ on $B$.

Proof. Let $f$ be a linear functional on $B$, and let $\theta_{n}=-\arg f\left(p_{n}\right)$.

$$
\sum_{n=0}^{k}\left|f\left(p_{n}\right)\right|=\sum_{n=0}^{k} e^{i \theta_{n}} f\left(p_{n}\right)<H\|f\|,
$$

whence the result follows.

LEMMA 4. The necessary and sufficient conditions that $\gamma(a)=\sum_{k=0}^{\infty} g_{k}(a) c_{k}$ should tend to a finite limit as $a \rightarrow \infty$ whenever $\Sigma c_{k}$ is convergent are:

(i) $\sum_{k=0}^{\infty}\left|g_{k}(a)-g_{k+1}(a)\right| \leqslant M$ independently of $a>a^{\prime}$;

(ii) $\lim _{a \rightarrow \infty} g_{k}(a)=\beta_{k}$ for every fixed $k$.

The proof of this lemma may be found in [5].

LEMMA 5. A necessary and sufficient condition for $\sum a_{n} \varepsilon_{n}$ to be summable $\left(A, \lambda_{n}\right)$ whenever $\Sigma a_{n}$ is convergent is that $\Sigma\left|\Delta \varepsilon_{n}\right|<\infty$.

It may be remarked that if we take $\lambda_{n}=n$ we get the following result of Bosanquet [Proc. London Math. Soc. (2) 50 (1948), Lemma 9].

Lemma A. $A$ necessary and sufficient condition for $\sum a_{n} \varepsilon_{n}$ to be summable $(A)$ whenever $\Sigma a_{n}$ is convergent is that $\Sigma\left|\Delta \varepsilon_{n}\right|<\infty$.

Proof of Lemma 5. Since $\sum a_{n} \varepsilon_{n}$ is summable $\left(A, \lambda_{n}\right)$ we have

$$
\sum_{n=1}^{\infty} a_{n} \varepsilon_{n} e^{-\lambda_{n} / t} \text { is convergent for } t>0
$$

and

$$
\lim _{t \rightarrow \infty} \sum_{n=1}^{\infty} a_{n} \varepsilon_{n} e^{-\lambda_{n} / t} \text { exists. }
$$

Applying Lemma 4 we have

$$
\sum_{n=1}^{\infty}\left|\Delta\left(\varepsilon_{n} e^{-\lambda_{n} / t}\right)\right| \leqslant M \quad \text { independently of } t>t_{0} .
$$

This implies that $\sum_{n=1}^{\infty}\left|\Delta \varepsilon_{n}\right|<\infty$.

The proof of the sufficiency part of the lemma is immediate for $\Sigma a_{n}$ is convergent and $\left\{\varepsilon_{n}\right\}$ is a sequence of bounded variation.

LEMMA 6. We have

$$
\int_{0}^{\infty}\left|d \Delta e^{-\lambda_{n} x}\right|=2\left(1-\mu_{n}\right)\left(\mu_{n}\right)^{\mu_{n} /\left(1-\mu_{n}\right)}
$$

The proof is simple. 
LEMMA 7. If $\mu_{n}=\lambda_{n} / \lambda_{n+1}$, then the following conditions are equivalent.

(i) $\Sigma\left|\varepsilon_{n}\right|\left(1-\mu_{n}\right)<\infty$,

(ii) $\Sigma\left|\varepsilon_{n}\right|\left(1-\mu_{n}\right) \mu_{n}^{\mu_{n} /\left(1-\mu_{n}\right)}<\infty$.

Proof. Since $0<\mu_{n}<1$, so $e^{-1}<\mu_{n}^{\mu_{n} /\left(1-\mu_{n}\right)}<1$. Hence (i) and (ii) are equivalent.

LEMMA 8. Let $M, a$ be constants with $M>1, a>0$ and

(i) $a \log M<2 \pi$.

Then for $1 / M \leqslant u<1$ we have $\left|1-u^{-a i}\right| /(1-u) \geqslant C$, where $C>0$ is a constant (depending on $a$ and $M$ ).

Proof. Given (i), the function $\left(1-u^{-a i}\right)(1-u)^{-1}$ is continuous and nonzero on $1 / M<u<1$, and has a nonzero limit as $u \rightarrow 1$. This proves the lemma.

LEMMA 9. For $k=1,\left|R, \lambda_{n}, k\right| \sim\left|R^{*}, \lambda_{n}, k\right|$.

Proof. Let

$$
\gamma(t)=t^{-k} \sum_{\lambda_{n}<t}\left(t-\lambda_{n}\right)^{k} a_{n}
$$

$\left|R, \lambda_{n}, k\right|$ means that

$$
\int_{0}^{\infty}|d \gamma(t)|<\infty
$$

$\left|R^{*}, \lambda_{n}, k\right|$ means

$$
\sum_{n=1}^{\infty}\left|\gamma\left(\lambda_{n}\right)-\gamma\left(\lambda_{n+1}\right)\right|<\infty
$$

Now if $k=1$, then for $\lambda_{n} \leqslant t \leqslant \lambda_{n+1}$,

$$
\gamma(t)=\frac{1}{t} \sum_{\nu=0}^{n}(t-\lambda \nu) a_{\nu}=A-B / t,
$$

where $A=\sum_{\nu=0}^{n} a_{\nu}, B=\sum_{\nu=0}^{n} \lambda_{\nu} a_{\nu}$. Thus, for a given $n, A, B$ are constants (though their values will vary with $n$ ).

Hence, for a given $n, \gamma(t)$ is monotonic in each interval $\left[\lambda_{n}, \lambda_{n+1}\right]$ (increasing if $B>0$ and decreasing if $B<0$ ). Hence

$$
\int_{\lambda_{n}}^{\lambda_{n+1}}|d \gamma(t)|=\left|\gamma\left(\lambda_{n+1}\right)-\gamma\left(\lambda_{n}\right)\right|
$$

so that (i) and (ii) are equivalent.

Note. It is known [6] that $\left|R, \lambda_{n}, k\right| \sim\left|R^{*}, \lambda_{n}, k\right|$ for $0<k<1$.

LEMMA 10. For any $k>0,\left|R, \lambda_{n}, k\right| \Rightarrow\left|A, \lambda_{n}\right|$.

The proof of this lemma may be found in [7].

\section{Proof of the theorem.}

Sufficiency. Since $\sum a_{n}$ is convergent, by virtue of (2.3) and Abel's test we see that $\sum a_{n} \varepsilon_{n} e^{-\lambda_{n} x}$ is convergent for all positive $x$, so that writing 


$$
\alpha(x)=\sum_{n=0}^{\infty} a_{n} \varepsilon_{n} e^{-\lambda_{n} x}
$$

we have

$$
\begin{aligned}
\alpha(x) & =\sum_{n=0}^{\infty} S_{n} \Delta\left(\varepsilon_{n} e^{-\lambda_{n} x}\right), \quad S_{n}=a_{0}+a_{1}+\cdots+a_{n} \\
& =\sum_{n=0}^{\infty} S_{n} e^{-\lambda_{n+1} x} \Delta \varepsilon_{n}+\sum_{n=0}^{\infty} S_{n} \varepsilon_{n} \Delta e^{-\lambda_{n} x} \\
& =\alpha_{1}(x)+\alpha_{2}(x) \quad \text { (say). }
\end{aligned}
$$

In order to prove that $\Sigma a_{n} \varepsilon_{n}$ is summable $\left|A, \lambda_{n}\right|$ it is sufficient to show that $\alpha_{1}(x)$ and $\alpha_{2}(x)$ are functions of bounded variation on $[0, \infty)$, that is to say,

$$
\int_{0}^{\infty}\left|d \alpha_{1}(x)\right|<\infty
$$

and

$$
\int_{0}^{\infty}\left|d \alpha_{2}(x)\right|<\infty
$$

Now

$$
\begin{aligned}
\int_{0}^{\infty}\left|d \alpha_{1}(x)\right| & =\int_{0}^{\infty}\left|d \sum_{n=0}^{\infty} S_{n} e^{-\lambda_{n+1} x} \Delta \varepsilon_{n}\right| \\
& <\sum_{n=0}^{\infty}\left|S_{n}\right| \cdot\left|\Delta \varepsilon_{n}\right| \int_{0}^{\infty}\left|d e^{-\lambda_{n+1} x}\right| \\
& <\overline{\mathrm{bd}}\left|S_{n}\right| \sum_{n=0}^{\infty}\left|\Delta \varepsilon_{n}\right|<\infty
\end{aligned}
$$

Also by virtue of Lemmas 6 and 7 and condition (2.4) we observe that

$$
\begin{aligned}
\int_{0}^{\infty}\left|d \alpha_{2}(x)\right| & =\int_{0}^{\infty}\left|d \sum_{n=0}^{\infty} S_{n} \varepsilon_{n} \Delta e^{-\lambda_{n} x}\right| \\
& <\sum_{n=0}^{\infty}\left|S_{n}\right| \cdot\left|\varepsilon_{n}\right| \int_{0}^{\infty}\left|d \Delta e^{-\lambda_{n} x}\right| \\
& <2 \overline{\mathrm{bd}}\left|S_{n}\right| \sum_{n=0}^{\infty}\left|\varepsilon_{n}\right|\left(1-\mu_{n}\right) \mu_{n}^{\mu_{n} /\left(1-\mu_{n}\right)} \\
& =2 \overline{\mathrm{bd}}\left|S_{n}\right| \sum_{n=0}^{\infty}\left|\varepsilon_{n}\right|\left(1-\mu_{n}\right)<\infty .
\end{aligned}
$$

This completes the sufficiency part of the theorem.

Necessity. Since summability $\left|A, \lambda_{n}\right|$ implies summability $\left(A, \lambda_{n}\right)$, it follows from Lemma 5 that (2.3) is necessary. Also from (4.1), since (2.3) holds, we have, as before, $\int_{0}^{\infty}\left|d \alpha_{1}(x)\right|<\infty$.

Since by hypothesis $\alpha(x)$ is of bounded variation it follows that $\alpha_{2}(x)$ is also of bounded variation. Therefore 


$$
\begin{aligned}
\int_{0}^{\infty}\left|\sum_{n=0}^{\infty} S_{n} \varepsilon_{n}\left[\frac{d}{d x} \Delta e^{-\lambda_{n} x}\right]\right| d x & =\int_{0}^{\infty}\left|\frac{d}{d x} \sum_{n=0}^{\infty} S_{n} \varepsilon_{n} \Delta e^{-\lambda_{n} x}\right| d x \\
& =\int_{0}^{\infty}\left|d \sum_{n=0}^{\infty} S_{n} \varepsilon_{n} e^{-\lambda_{n} x}\right|<\infty
\end{aligned}
$$

for every convergent sequence $\left\{S_{n}\right\}$. Applying Lemma 2 we have

$$
\int_{0}^{\infty}\left|\sum_{n=0}^{\infty} S_{n} \varepsilon_{n}\left[\frac{d}{d x} \Delta e^{-\lambda_{n} x}\right]\right| d x \leqslant H \overline{\mathrm{bd}}\left|S_{n}\right|
$$

for every sequence $\left\{S_{n}\right\}$. In particular, we have

$$
\int_{0}^{\infty}\left|\sum_{n=0}^{k} e^{i \theta_{n}} \varepsilon_{n}\left[\frac{d}{d x} \Delta e^{-\lambda_{n} x}\right]\right| d x<H
$$

for every nonnegative integer $k$ and every set of real numbers $\theta_{0}, \theta_{1}, \ldots, \theta_{k}$. Again the sequence $\left\{\varepsilon_{n}(d / d x) \Delta e^{-\lambda_{n} x}\right\} \in L$, and so

$$
\left\|\sum_{n=0}^{k} e^{i \theta_{n}} \varepsilon_{n} \frac{d}{d x} \Delta e^{-\lambda_{n} x}\right\| \leqslant H,
$$

by virtue of inequality (4.5). Hence, by Lemma 3 we have

$$
\sum_{n=0}^{\infty}\left|f\left(\varepsilon_{n} \frac{d}{d x} \Delta e^{-\lambda_{n} x}\right)\right|<\infty
$$

for every linear functional $f$ on $L$.

But, for a given bounded measurable complex function $\phi(x)$,

$$
f_{\phi}(\psi)=\int_{0}^{\infty} \phi(x) \psi(x) d x
$$

is a linear functional on $L$. Therefore

$$
\sum_{n=0}^{\infty}\left|\int_{0}^{\infty} \phi(x) \varepsilon_{n} \frac{d}{d x} \Delta e^{-\lambda_{n} x} d x\right|<\infty
$$

This implies that

$$
\sum_{n=0}^{\infty}\left|\varepsilon_{n}\right|\left|\int_{0}^{\infty} \phi(x) \frac{d}{d x} \Delta e^{-\lambda_{n} x} d x\right|<\infty .
$$

Since $\lambda_{n+1}=O\left(\lambda_{n}\right)$ so there exists some constant $M$ such that, for all $n>1$, $\lambda_{n+1}<M \lambda_{n}$. Given this $M$, choose $a>0$ so that $a \log M<2 \pi$. Then apply (4.6) with $\phi(x)=x^{a i}$. (This choice is suggested by the argument of [3].) Now

$$
\begin{aligned}
\int_{0}^{\infty} \phi(x) d\left(\Delta e^{-\lambda_{n} x}\right) & =\Gamma(1+i a)\left(-\lambda_{n}^{-a i}+\lambda_{n+1}^{-a i}\right) \\
& =\Gamma(1+i a) \lambda_{n+1}^{-a i}\left(1-\mu_{n}^{-a i}\right) .
\end{aligned}
$$

But, for sufficiently large $n, \mu_{n} \geqslant 1 / M$. Hence by Lemma 8 ,

$$
\left|\int_{0}^{\infty} \phi(x) d\left(\Delta e^{-\lambda_{n} x}\right)\right| \geqslant C|\Gamma(1+i a)|\left(1-\mu_{n}\right) .
$$


It therefore follows from (4.6) that $\sum_{n=0}^{\infty}\left|\varepsilon_{n}\right|\left(1-\mu_{n}\right)<\infty$ as required.

5. The sufficiency part of the theorem can be improved. If (2.3) and (2.4) hold, then, in fact, $\sum_{n=0}^{\infty} a_{n} \varepsilon_{n}$ is summable $\left|R, \lambda_{n}, 1\right|$ whenever $\sum_{n=0}^{\infty} a_{n}$ is convergent. By Lemma 10 this is stronger than the present result. For summability $\left|R, \lambda_{n}, 1\right|$ implies summability $\left|A, \lambda_{n}\right|$ when the $\left(A, \lambda_{n}\right)$ method is applicable, i.e., whenever

$$
\sum_{n=0}^{\infty} a_{n} \varepsilon_{n} e^{-\lambda_{n} x}
$$

converges for all $x>0$. But (2.3) alone is enough to ensure that whenever $\sum_{n=0}^{\infty} a_{n}$ converges, $\sum_{n=0}^{\infty} a_{n} \varepsilon_{n}$ converges, and thus (5.1) certainly converges.

Hence our Theorem 1 can be improved in the following way.

Theorem 2. Conditions (2.3) and (2.4) are sufficient for $\sum_{n=0}^{\infty} a_{n} \varepsilon_{n}$ to be summable $\left|R, \lambda_{n}, 1\right|$ whenever $\sum_{n=0}^{\infty} a_{n}$ converges, and necessary for it to be summable $\left|A, \lambda_{n}\right|$ whenever $\sum_{n=0}^{\infty} a_{n}$ converges and $\lambda_{n+1}=O\left(\lambda_{n}\right)$.

Proof. In the light of Theorem 1 , it is sufficient to show that $\sum_{n=0}^{\infty} a_{n} \varepsilon_{n}$ is summable $\left|R, \lambda_{n}, 1\right|$ whenever $\sum_{n=0}^{\infty} a_{n}$ converges. To prove our assertion it is enough, by Lemma 9, to consider the discontinuous Riesz means, so write

$$
t_{n}=-\frac{1}{\lambda_{n+1}} \sum_{k=0}^{n}\left(\lambda_{n+1}-\lambda_{k}\right) a_{k} \varepsilon_{k} .
$$

Suppose that $\sum_{k=0}^{\infty} a_{k}$ converges and that (2.3) and (2.4) hold. Suppose without loss of generality that $a_{0}=0$. Then, for $n \geqslant 1$,

$$
\begin{aligned}
t_{n}-t_{n-1} & =\left(1 / \lambda_{n}-1 / \lambda_{n+1}\right) \sum_{k=1}^{n} \lambda_{k} a_{k} \varepsilon_{k} \\
& =\left(1 / \lambda_{n}-1 / \lambda_{n+1}\right)\left[\sum_{k=1}^{n-1} S_{k} \lambda_{k+1} \Delta \varepsilon_{k}+\sum_{k=1}^{n-1} S_{k} \varepsilon_{k} \Delta \lambda_{k}+S_{n} \varepsilon_{n} \lambda_{n}\right] \\
& =b_{n}+c_{n}+d_{n} \text { (say). }
\end{aligned}
$$

Supposing that for all $n,\left|S_{n}\right| \leqslant K$, we have

$$
\begin{gathered}
\sum_{n=1}^{\infty}\left|b_{n}\right| \leqslant K \sum_{k=1}^{\infty}\left|\Delta \varepsilon_{k}\right| \lambda_{k+1} \sum_{n=k+1}^{\infty}\left(1 / \lambda_{n}-1 / \lambda_{n+1}\right) \\
=K \sum_{k=1}^{\infty}\left|\Delta \varepsilon_{k}\right|<\infty \quad \text { by }(2.3) \\
\sum_{n=1}^{\infty}\left|c_{n}\right| \leqslant K \sum_{k=1}^{\infty}\left|\varepsilon_{k}\right|\left|\Delta \lambda_{k}\right| \sum_{n=k+1}^{\infty}\left(1 / \lambda_{n}-1 / \lambda_{n+1}\right) \\
=K \sum_{k=1}^{\infty}\left|\varepsilon_{k}\right|\left(\lambda_{k+1}-\lambda_{k}\right) / \lambda_{k+1} \\
=K \sum_{k=1}^{\infty}\left|\varepsilon_{k}\right|\left(1-\lambda_{k} / \lambda_{k+1}\right)=K \sum_{k=1}^{\infty}\left|\varepsilon_{k}\right|\left(1-\mu_{k}\right)<\infty \quad \text { by (2.4). }
\end{gathered}
$$




$$
\begin{aligned}
\sum_{n=1}^{\infty}\left|d_{n}\right| & <K \sum_{n=1}^{\infty}\left|\varepsilon_{n}\right| \lambda_{n}\left(1 / \lambda_{n}-1 / \lambda_{n+1}\right) \\
& =K \sum_{n=1}^{\infty}\left|\varepsilon_{n}\right|\left(1-\lambda_{n} / \lambda_{n+1}\right)=K \sum_{n=1}^{\infty}\left|\varepsilon_{n}\right|\left(1-\mu_{n}\right) \\
& <\infty \text { by }(2.4) .
\end{aligned}
$$

Hence the result.

Acknowledgement. The author is grateful to Professor S. M. Mazhar, University of Kuwait, for his kind help in the preparation of this paper.

\section{REFERENCES}

1. G. H. Hardy, Divergent series, Oxford Univ. Press, London, 1949.

2. M. W. Orlicz, Beitrage zur Theorie der Orthogonalentwicklungen. II, Studia Math. 1 (1929), 241-255.

3. J. B. Tatchell, A note on a theorem by Basanquet, J. London Math. Soc. 29 (1954), $207-211$.

4. , On some integral transforms, Proc. London Math. Soc. 3 (1953), 257-267.

5. P. Dienes, The Taylor series, Dover, New York, 1957, p. 396.

6. H. H. Körle, Über Unsteige absolute Riesz-Summujung. I, II, Math. Ann. 176 (1968), 45-52; ibid. 177 (1968), 230-234.

7. J. S. Ratti, Tauberian theorems for absolute summability, Proc. Amer. Math. Soc. 18 (1967), 775-781.

DePARTMENT OF MATHEMATICS, KURUKSHETRA UNIVERSTTY, KURUKSHETRA-132119, INDIA 